\title{
Dexmedetomidine for chronic spinal cord injured patient
}

\author{
Ki Hwa Lee
}

Received: 10 March 2014/ Accepted: 18 March 2014/Published online: 6 April 2014

(C) Japanese Society of Anesthesiologists 2014

Keywords Dexmedetomidine $\cdot$ Monitored anesthesia care $\cdot$ Spinal cord injury

To the Editor:

Anesthesia for spinal cord injured (SCI) patients is challenging for anesthesiologists. Although many patients undergoing surgery below the injured spinal cord level can proceed without anesthesia, autonomic dysreflexia (AD) and spasms can occur, and patients often require adequate sedation and anxiolysis during the operation. $\mathrm{AD}$ can occur not only in patients with lesions above T6, but also in those with lower lesion levels [1]. Therefore, close monitoring and careful management is essential for SCI patients [2].

We experienced a case of a 66-year-old man with a history of paraplegia. He had lost sensory and motor response below the $\mathrm{T} 7$ level because of a traffic accident 40 years earlier. The patient preferred to be unconscious for surgery. After monitoring vital signs, dexmedetomidine was infused at a rate of $0.6 \mu \mathrm{g} / \mathrm{kg} / \mathrm{h}$ for monitored anesthesia care (MAC). No AD or spasm symptoms occurred, and the patient's BIS was maintained with moderate sedation (70-80). Open bladder stone removal was done successfully without anesthesia.

Hypertensive crisis induced by AD is paroxysmal and may be dangerous. Patients with SCI are sensitive to circulating noradrenaline [2]. Dexmedetomidine, a highly selective $\alpha_{2}$-adrenergic agonist, reduces circulating catecholamines and results in sympatholysis, anxiolysis, and respiratory preservation [3]. Thus, dexmedetomidine can be used as a safe sedative agent during MAC in patients with chronic SCI.

Conflict of interest There are no conflicts of interest.

\section{References}

1. Huang YH, Bih LI, Chen GD, Lin CC, Chen SL, Chen WW. Autonomic dysreflexia during urodynamic examinations in patients with suprasacral spinal cord injury. Arch Phys Med Rehabil. 2011;92:1450-4.

2. Hambly PR, Martin B. Anaesthesia for chronic spinal cord lesions. Anaesthesia. 1998;53:273-89.

3. Arcangeli A, D'Alò C, Gaspari R. Dexmedetomidine use in general anaesthesia. Curr Drug Targets. 2009;10:687-95.
K. H. Lee $(\bowtie)$

Haeundae Paik Hospital, Busan, Republic of Korea

e-mail: tedy333@paik.ac.kr 